\title{
Coherent Polariton Laser
}

\author{
Seonghoon Kim, ${ }^{1}$ Bo Zhang, ${ }^{2}$ Zhaorong Wang, ${ }^{1}$ Julian Fischer, ${ }^{3}$ Sebastian Brodbeck, ${ }^{3}$ Martin Kamp, ${ }^{3}$ \\ Christian Schneider, ${ }^{3}$ Sven Höfling, ${ }^{3,4}$ and Hui Deng ${ }^{2, *}$ \\ ${ }^{1}$ Department of Electrical Engineering and Computer Science, University of Michigan, \\ 1301 Beal Avenue, Ann Arbor, Michigan 48109-2122, USA \\ ${ }^{2}$ Department of Physics, University of Michigan, 450 Church Street, \\ Ann Arbor, Michigan 48109-1040, USA \\ ${ }^{3}$ Technische Physik, Universität Würzburg, Am Hubland, D-97074 Würzburg, Germany \\ ${ }^{4}$ SUPA, School of Physics and Astronomy, University of St. Andrews, \\ St. Andrews KY16 9SS, United Kingdom
}

(Received 25 July 2015; revised manuscript received 1 December 2015; published 11 March 2016)

\begin{abstract}
The semiconductor polariton laser promises a new source of coherent light, which, compared to conventional semiconductor photon lasers, has input-energy threshold orders of magnitude lower. However, intensity stability, a defining feature of a coherent state, has remained poor. Intensity noise many times the shot noise of a coherent state has persisted, attributed to multiple mechanisms that are difficult to separate in conventional polariton systems. The large intensity noise, in turn, limits the phase coherence. Thus, the capability of the polariton laser as a source of coherence light is limited. Here, we demonstrate a polariton laser with shot-noise-limited intensity stability, as expected from a fully coherent state. This stability is achieved by using an optical cavity with high mode selectivity to enforce single-mode lasing, suppress condensate depletion, and establish gain saturation. Moreover, the absence of spurious intensity fluctuations enables the measurement of a transition from exponential to Gaussian decay of the phase coherence of the polariton laser. It suggests large self-interaction energies in the polariton condensate, exceeding the laser bandwidth. Such strong interactions are unique to matter-wave lasers and important for nonlinear polariton devices. The results will guide future development of polariton lasers and nonlinear polariton devices.
\end{abstract}

DOI: 10.1103/PhysRevX.6.011026

\section{INTRODUCTION}

Coherent light, characterized by long phase coherence and shot-noise-limited intensity stability [1], has been a revolutionary resource in modern science and technology. The coherence in conventional photon lasers is formed through the stimulated photon emission process that requires population inversion in the incoherent gain medium, which sets a lower bound for the energy threshold. In contrast, a coherent matter wave can form as the ground state, rather than the inverted state, of a many-body quantum system. Therefore, semiconductor polaritons, half-light and halfmatter quasiparticles that can form a condensate [2], promise an energy-efficient source of coherent light without electronic population inversion $[3,4]$, called the polariton laser.

Polariton condensation and lasing at a low threshold density have been demonstrated worldwide in diverse

\footnotetext{
*Corresponding author. dengh@umich.edu

Published by the American Physical Society under the terms of the Creative Commons Attribution 3.0 License. Further distribution of this work must maintain attribution to the author(s) and the published article's title, journal citation, and DOI.
}

Subject Areas: Condensed Matter Physics, Photonics, Quantum Physics materials [4-10] under both optical and electrical excitations [11-13]. Evidence of polariton lasing in the polariton ground state includes quantum degeneracy [14], reduced intensity fluctuations compared to thermal states [14], increased spatial [15] and temporal [16] coherence, and spontaneous polarization buildup [17].

In these polariton lasers, however, a significant fraction of thermal population was either clearly present in the ground state or could not be excluded. Consequently, phase coherence and intensity stability, the defining features of a coherent state that are crucial for many applications of lasers, have been limited. The intensity noise is measured by the second-order coherence function $g^{(2)}(0)$ :

$$
g^{(2)}(0)=1+\frac{\sigma_{n}^{2}-\bar{n}}{\bar{n}^{2}},
$$

where $\sigma_{n}^{2}$ is the variance of the photon number and $\bar{n}$ is the average photon number. For a coherent state with Poisson number statistics, $\sigma_{n}^{2}=\bar{n}$ and $g^{(2)}(0)=1$, as has been observed in both photon lasers and coherent matter waves of atoms [18-20]. In polariton lasers, however, large intensity noise has persisted, as measured by $g^{(2)}(0)>1$ $[14,16,21-25]$. One of the well-calibrated values reached as low as $g^{(2)}(0)=1.1$, which still corresponds to a variance $\sigma_{n}^{2}$ that is 50 times the shot-noise limit [16]. Such large 
intensity noise in polariton lasers has been attributed to many possible mechanisms, including mode competition among multiple spatial modes or momentum states [26], reservoir-induced intensity fluctuations [27], and condensate depletion $[28,29]$. These different mechanisms coexisted in previous experiments and could not be separately identified or controlled. Agreements between experiments and theory were qualitative. No solution has been clearly identified to improve the intensity stability of a polariton laser. The quantum statistical nature of polariton lasers and their potential use as a source of coherent light are unclear.

The spurious intensity fluctuations, in turn, limit the phase coherence. The phase coherence is described by the first-order coherence function $g^{(1)}(\tau)$, which equals 1 at $\tau=0$ and decays with a coherence time corresponding to the inverse linewidth of the light. In a conventional photon laser, $g^{(1)}(\tau)$ decays exponentially, and the coherence time increases proportionally to the photon occupation number, giving the Schawlow-Townes linewidth [30]. For polariton lasers, however, the coherence time is limited by energy fluctuations induced by pulsed excitation in early experiments. When intensity-stabilized excitation sources are used, energy fluctuations resulting from the spurious intensity fluctuations of the condensate become a main dephasing mechanism [16]. Consequently, the intrinsic limit of phase coherence of the polariton laser remains obscured.

In this work, we demonstrate a coherent polariton laser with intensity and phase noise limited only by the intrinsic shot noise of the condensate. We achieve intensity stability at the Poisson limit in a single-mode polariton laser with suppressed mode competition and condensate depletion by a cavity with high mode selectivity. Gain saturation was established to avoid intensity fluctuations induced by the reservoir and nonlasing modes. With full intensity stability, the intrinsic phase coherence of the polariton laser showed a transition from the Schawlow-Townes limit at low condensate occupancies to Gaussian dephasing at high occupancies. Such a transition was predicted for matter-wave lasers but not observed in experiments before. The Gaussian dephasing results from strong interactions within the condensate, which is unique to matter-wave lasers $[31,32]$ and important for nonlinear polariton devices [33-35].

\section{CAVITY SYSTEM}

The cavity used in our work has both high polarization selectivity and tight lateral confinement, so as to suppress mode competition and condensate depletion while enhancing condensate nonlinearity. As illustrated in Fig. 1(a), a suspended high-index-contrast subwavelength grating (SWG) is used as the top mirror [36], in place of a more commonly used distributed Bragg reflector (DBR). The grating breaks the rotational symmetry and is designed to allow high reflectance for only the transverse-electric (TE) polarized mode [36,37]. Hence, polariton modes were formed in a single, predetermined spin state [38]. This eliminates mode competition between different spin states, which is ubiquitous in DBR-DBR cavities. The high index contrast allows high reflectance even with SWGs of a few wavelengths in size. Hence, polariton modes are tightly confined to within the SWG region of $7.5 \mu \mathrm{m} \times 7.5 \mu \mathrm{m}$ [38,39], featuring a discrete energy spectrum [Fig. 1(b)]. The nondegenerate ground state is separated from the first excited state by about $1 \mathrm{meV}$. The energy gap protects the condensate from quantum depletion and mode competition with the excited states. In addition, the discrete energy levels allow us to unambiguously select and measure each individual state, as illustrated in Fig. 1(c). The tight
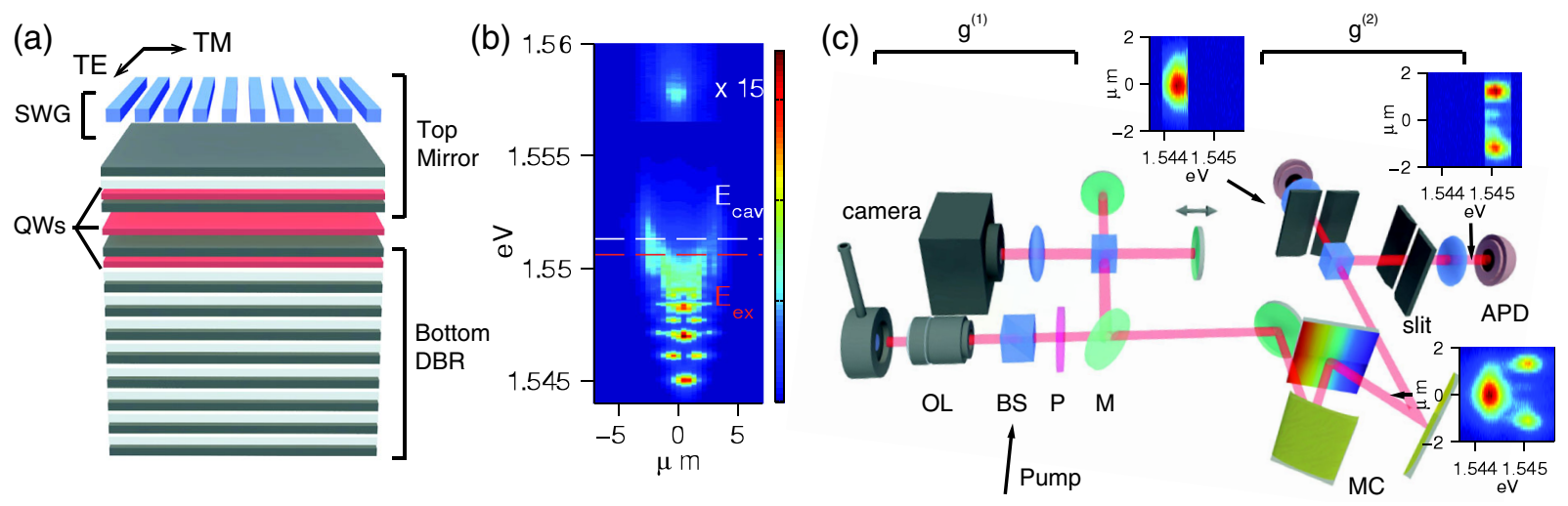

FIG. 1. The cavity system and experimental setup. (a) A schematic of the SWG-based microcavity. (b) The spectrally resolved realspace image of polariton states at a low excitation power. $\mathrm{E}_{\mathrm{cav}}$ is the cavity resonance, and $\mathrm{E}_{\mathrm{ex}}$ is the exciton resonance. $\mathrm{E}_{\mathrm{cav}}$ is estimated by using lower polariton, upper polariton, and exciton energies. (c) A schematic of the experimental setup. OL: objective lens; BS: beam splitter, P: polarizer; M: mirror; MC: monochromator; and APD: avalanche photodetector. For the $g^{(2)}$ measurements, a monochromator followed by two mechanical slits was used to spectrally filter the discrete polariton states. Examples are shown for the spectrally resolved real-space images of the lowest two LP states right after the monochromator (bottom), the spectrally filtered ground state (top left), and the spectrally filtered first excited state (top right). The resolution of the spectral filter was about $0.08 \mathrm{~nm}$, determined by the monochromator resolution. 
confinement also enhances the nonlinear polaritonpolariton interactions.

\section{SPECTRAL PROPERTIES OF THE POLARITON LASER}

With increasing excitation density $P$, polariton lasing is observed by a sharp superlinear increase of the ground-state population $\bar{n}$ around the threshold $P_{\text {th }}$ [Figs. 2(a) and 2(d)], corresponding to the onset of quantum degeneracy in the ground state, or $\bar{n}=1$. At the same time, the spectral linewidth of the ground-state polariton emission narrowed, as shown in Figs. 2(c) and 2(f), reflecting increased phase coherence. The accurate linewidth and coherence time of the polariton laser were measured by a Michelson interferometer with continuous-wave $(\mathrm{CW})$ excitation, as we discuss later.

Additional confirmation of polariton lasing, rather than photon lasing, was shown by the emission energy $E_{\mathrm{LP}}$ of the polariton laser vs $P$ in Figs. 2(b) and 2(d). The energy of the polariton laser corresponds to the resonance energy of the polariton ground state; hence, it blueshifts with increasing $P$ because of the phase-space filling and polariton interactions. Moreover, the blueshift is continuous across the polariton lasing threshold, and $E_{\mathrm{LP}}$ remains well below the cavity or exciton resonances up to many times above the threshold, confirming that the strong-coupling regime is maintained.

In contrast, drastically different spectral properties are observed when a transition to photon lasing in the weakcoupling regime takes place at $P \sim 6 P_{\text {th }}$ under pulsed excitations. The transition is marked by a sudden appearance of a new lasing mode pinned at the energy of the cavity resonance with a sharply decreased linewidth [red symbols in Figs. 2(b) and 2(c)].

\section{INTENSITY NOISE OF THE POLARITON LASER}

We characterize the intensity noise of the polariton laser by measurements of $g^{(2)}(\tau)$ using a Hanbury Brown-Twiss (HBT) type of setup [Fig. 1(c)], under both pulsed and CW excitations. The measured value $\overline{g^{(2)}}(0)$ is an integration of
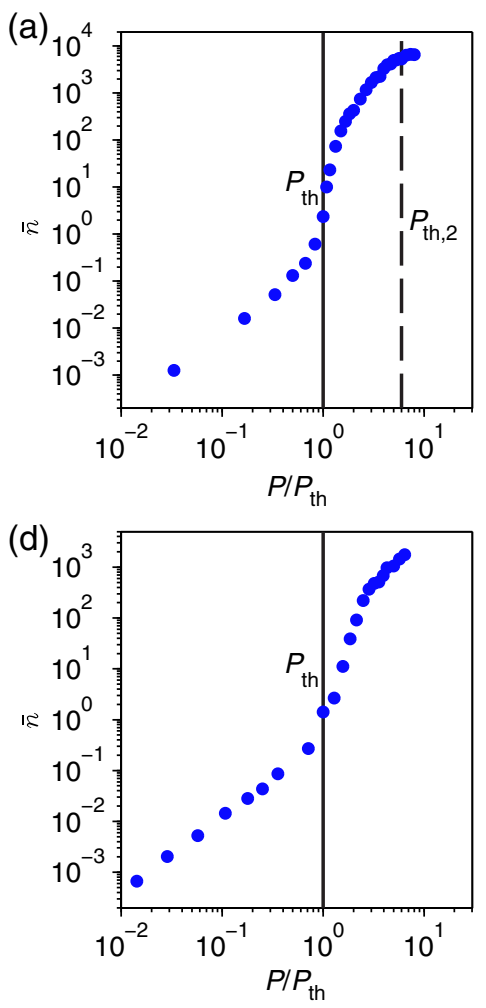

(b)

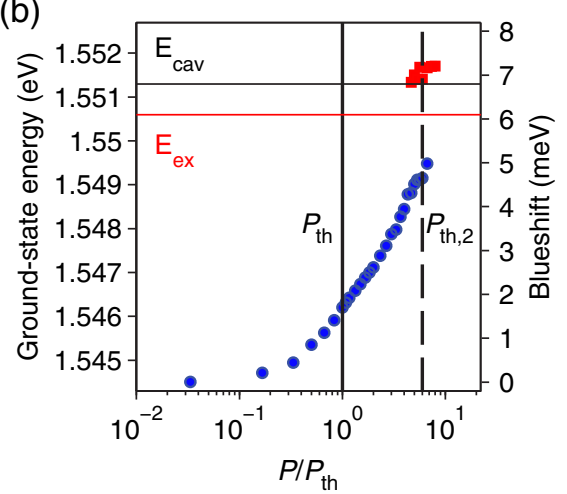

(e)

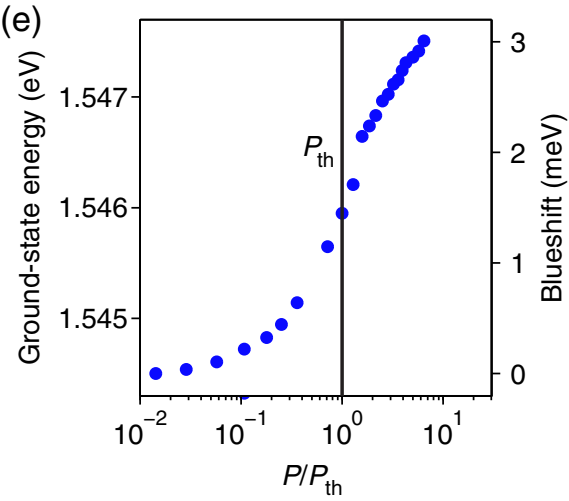

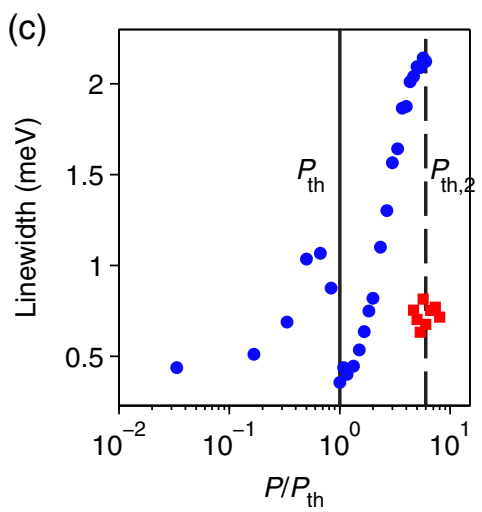

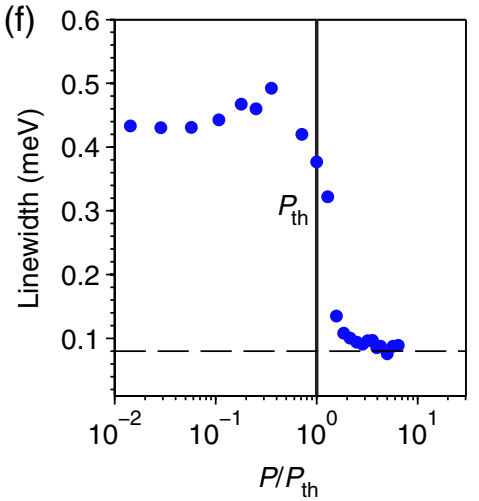

FIG. 2. Intensity and spectral properties of the single-mode polariton laser. (a) The occupation number vs $P / P_{\text {th }}$ for pulsed excitation. $P_{\text {th }}$ indicates the threshold for polariton lasing, and $P_{\text {th }, 2}$ indicates the threshold for photon lasing. The $\bar{n}$ is estimated from the independently measured PL intensity from the ground state, collection and detection efficiencies of the setup, and the polariton lifetime [4,6]. (b) Energy and blueshift of the polariton ground state (dots) and lasing photon mode (squares) vs the normalized pump powers $P / P_{\text {th }}$ for pulsed excitation. Here, $P_{\text {th }}$ is the pump power at the polariton lasing threshold. The solid vertical line marks the polariton lasing threshold, and the dashed vertical line marks the photon lasing threshold. (c) The linewidth (full width at half maximum) of the polariton ground state vs $P / P_{\text {th }}$ for pulsed excitation. (d)-(f) The occupation number, blueshift, and linewidth of the polariton ground state vs $P / P_{\text {th }}$ for $\mathrm{CW}$ excitation. The dashed line in (f) represents the spectral resolution of the monochromator of about $0.08 \mathrm{~nm}$. 
$g^{(2)}(\tau)$ over the measurement time $\Delta T . \Delta T$ is the duration of the emission pulse for pulsed excitation or the detector time resolution of $40 \mathrm{ps}$ for $\mathrm{CW}$ excitation. For a singlemode polariton laser, the $g^{(2)}(\tau)$ vs $\tau$ relation is known. Therefore, the actual deviation from the shot-noise limit, $\left|g^{(2)}(0)-1\right|$, or its upper bound can be obtained accurately from $\overline{g^{(2)}}(0)$ [40].

Examples of the measured $\overline{g^{(2)}}(\tau)$ vs $\tau$ are shown in Figs. 3(a) and 3(b). For both pulsed and CW excitations, bunching is evident below threshold but absent above threshold, showing the transition to a coherent state above threshold. The variation of $\overline{g^{(2)}}(0)$ with the normalized excitation power $P / P_{\text {th }}$ is shown in Fig. 3(c), with the corresponding $g^{(2)}(0)$ vs $\bar{n}$ shown in the inset.
A rapid transition from a thermal to a coherent state is evident. Near $P_{\text {th }}$, where the ground-state occupation number $\bar{n}$ is small, bunching is measured with $\overline{g^{(2)}}(0)$ as high as $1.248 \pm 0.007$ under pulsed excitations, corresponding to $g^{(2)}(0) \sim 2$ after correcting for the time average [40]. With the onset of quantum degeneracy and a sharp increase of $\bar{n}$ with $P$, the intensity noise rapidly decreases toward the coherent limit. Between $2 P_{\text {th }}$ and $6 P_{\text {th }}$, with condensate occupation number $\bar{n}=10^{2}-10^{3}$, the measured and corrected values of intensity noise remain around unity, with $0.994 \pm 0.006 \leq \overline{g^{(2)}}(0) \leq 1.009 \pm 0.005$ $\left[0.988 \pm 0.012 \leq g^{(2)}(0) \leq 1.020 \pm 0.011\right]$, and the average intensity noise in this range is $\overline{g^{(2)}}(0)=1.002 \pm 0.002$ $\left[g^{(2)}(0)=1.004 \pm 0.004\right]$. These results demonstrate the
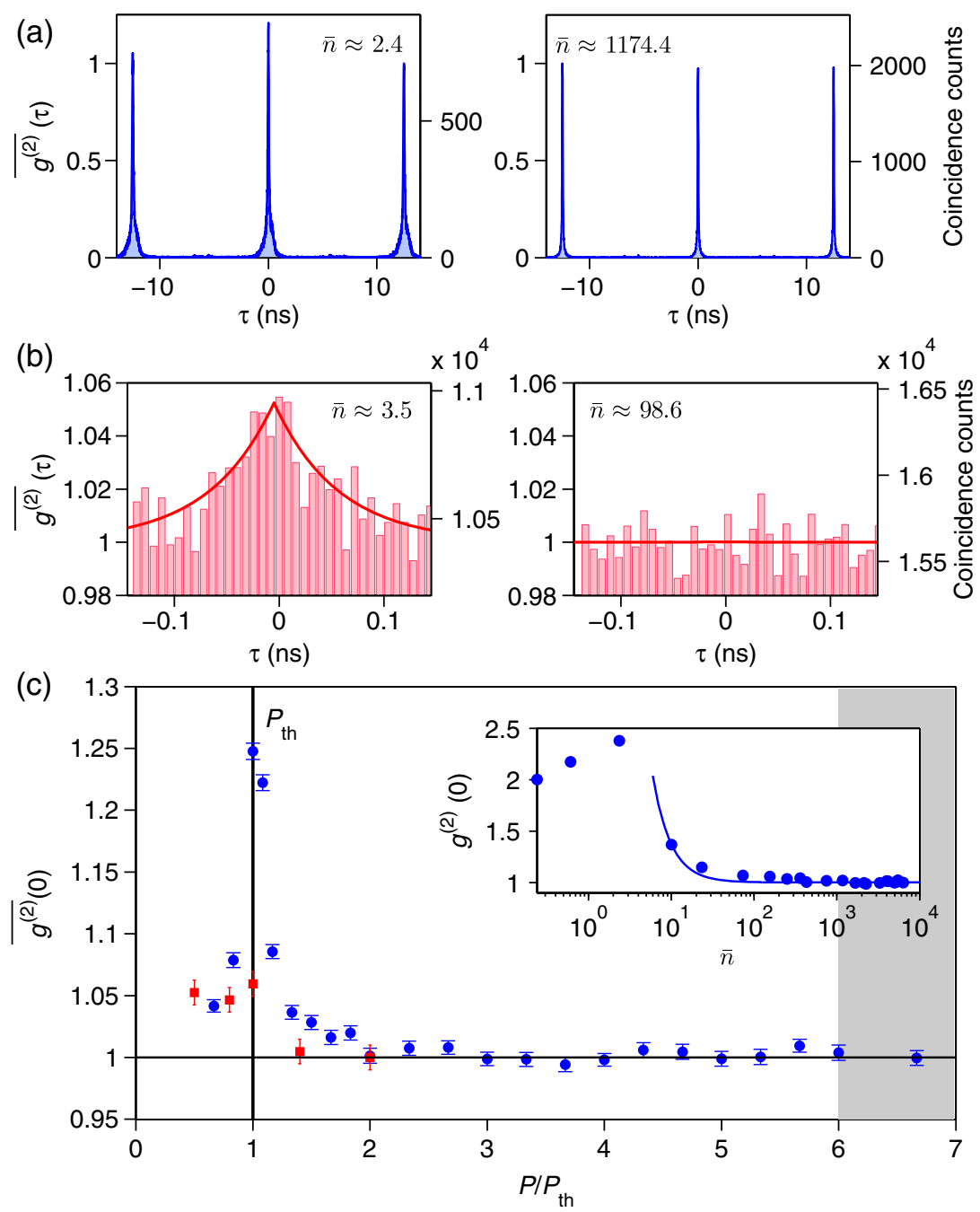

FIG. 3. Second-order coherence properties of the single-mode polariton laser. (a) $\overline{g^{(2)}}(\tau)$ vs $\tau$ below and above the threshold for pulsed excitation. (b) $\overline{g^{(2)}}(\tau)$ vs $\tau$ below and above the threshold for CW excitation. (c) $\overline{g^{(2)}}(0)$ vs $P / P_{\text {th }}$ for pulsed (dots) and CW (rectangles) excitations. The error bars indicate statistical error of 1 standard deviation. The grey-shaded area shows where the polariton and photon lasing coexist. Inset: $g^{(2)}(0)$ vs $\bar{n}$ of pulsed excitation corrected for the relaxation time of the ground state [40]. The solid line shows a theoretical fit by Eq. (2), yielding $n_{s}=37.3 \pm 0.9$. 
rapid formation of a coherent state with Poisson intensity noise in a polariton laser.

The experimental data are very well described by an analytical model for single-mode matter-wave lasers $[16,31,32,41]$. The model includes the interaction within the lasing mode or, in our system, the self-interaction among the condensed polaritons. It also includes other essential mechanisms of a laser: gain, gain saturation, and decay of the lasing mode. In the Bose-degenerate limit of $\bar{n} \gg 1, g^{(2)}(0)$ can be obtained as $[31,41]$

$$
g^{(2)}(0)=1+\frac{n_{s}}{\bar{n}^{2}},
$$

where $n_{s}$ is the gain saturation number. Comparing Eq. (2) and Eq. (1), we see that the total number fluctuations in the condensate is $\sigma_{n}^{2}=n_{s}+\bar{n} ; n_{s}$ represents fluctuations induced by the reservoir and other noncondensed modes, while $\bar{n}$ represents the intrinsic shot noise of the condensate. The coherent limit is reached when $\bar{n} \gg \sqrt{n_{s}}$. Fitting our data with Eq. (2) gives $n_{s}=37.3 \pm 0.9$, with $g^{(2)}(0)-1<10^{-3}$ at $P>2.5 P_{\text {th }}$ [inset of Fig. 3(c)].

Our result is in sharp contrast to previous $2 \mathrm{D}$ polariton lasers. Previously, a slow decrease of $g^{(2)}(0)$ with $P$ toward a value above unity was commonly observed, suggesting large $n_{s}$. For example, in a 2D system featuring multiple localized lasing modes with long coherence times, $g^{(2)}(0)=1.1$ is obtained for the selected lasing mode with $\bar{n} \sim 500$. Correspondingly, $n_{s}=25,000 \gg \bar{n}$ dominates the intensity noise [16]. In other experiments, $g^{(2)}(0)$ is typically higher or could not be obtained accurately. Since a condensate population of $10^{2}-10^{4}$ is commonly reported when the transition to the weak-coupling regime takes place, a relatively small $n_{s}$ as shown here is crucial for establishing intensity stability in a polariton laser.

In addition to reservoir-induced intensity fluctuations, we show the effect of mode competition on $g^{(2)}(0)$ in a multimode polariton laser. We establish two-mode lasing in the same system under the same experimental conditions, except for moving the excitation laser spot from the center of the device to slightly off-center to increase its overlap with the first excited state of the polariton [6]. The input-output relationships of both lasing modes are shown in Fig. 4(a). Contrary to a single-mode laser, clear deviations of $g^{(2)}(0)$ from unity are observed for both the ground and first-excited states, with $\overline{g_{00}^{(2)}}(0)\left[g_{00}^{(2)}(0)\right]=1.023 \pm 0.009(1.048 \pm 0.019)$ and $\overline{g_{11}^{(2)}}(0)\left[g_{11}^{(2)}(0)\right]=1.027 \pm 0.009(1.057 \pm 0.019)$, respectively [Fig. 4(b)]. The increased intensity fluctuations can be explained by the stochastic relaxation of polaritons from a common reservoir into the lasing modes [26]. Consistent with this explanation, we measured strong anticorrelation between the two modes, as shown by a cross-correlation function $\overline{g_{12}^{(2)}}(0)<1$. We note that such mode competition is difficult to eliminate in $2 \mathrm{D}$ or quasi-2D systems because the linewidth

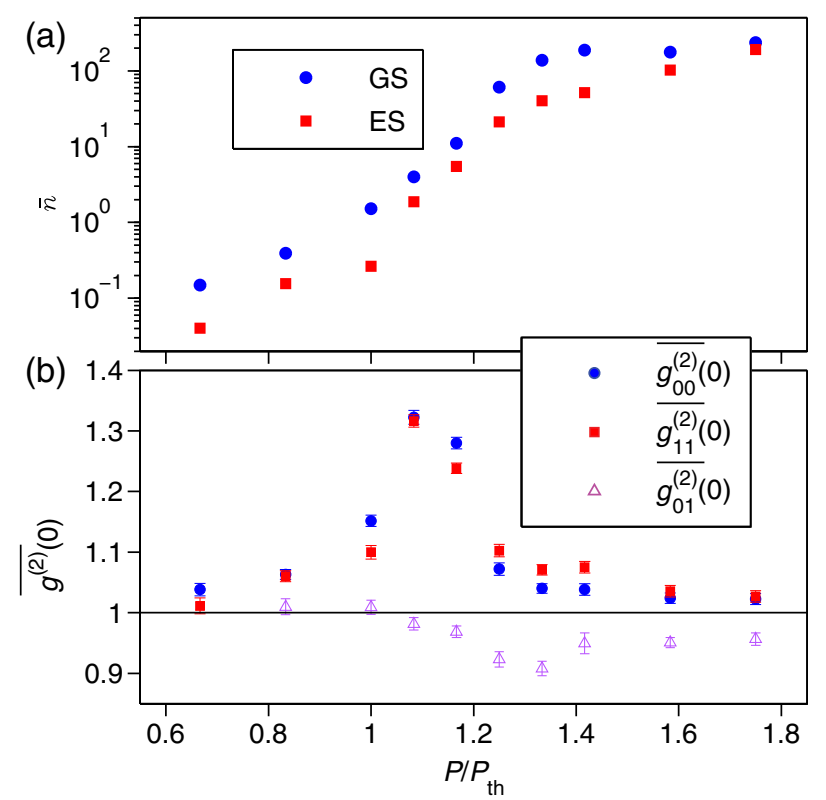

FIG. 4. Second-order correlations of the two-mode polariton laser. (a) The occupation numbers of the ground state (dots) and the excited state (squares) vs $P / P_{\text {th. }}$ GS: ground state; ES: first excited state. (b) $\overline{g^{(2)}}(0)$ vs $P / P_{\text {th }} \cdot \overline{g_{00}^{(2)}}(0)$ : autocorrelation of GS; $\overline{g_{11}^{(2)}}(0)$ : autocorrelation of ES; and $\overline{g_{01}^{(2)}}(0)$ : cross-correlation between GS and ES.

of the lasing mode is typically larger or comparable to the energy separation between LP modes of different polarizations, of different momenta, or in different localization potentials. Our results show that single-mode lasing, achieved by both tight lateral confinement and polarization selectivity in our system, is also crucial for intensity stability of a polariton laser.

\section{PHASE NOISE AND THE CONDENSATE INTERACTION ENERGY}

The temporal phase coherence of a polariton laser is described by the first-order correlation function $g^{(1)}(\tau)$. It is related to the power spectrum of the polariton emission by a Fourier transform. Using an intensity-stabilized CW laser for excitation, we measure $g^{(1)}(\tau)$ of the single-mode polariton laser using a Michelson interferometer [Fig. 1(c)]. The visibility of the interference fringes gives $g^{(1)}(\tau)$ (see the Appendix). Examples of the interference fringes are shown in Figs. 5(a) and 5(b). We then obtain $g^{(1)}(\tau)$ vs $\tau$ at different excitation powers by varying the delay time $\tau$ between the two arms of the interferometer.

Very different $\tau$ dependence of $g^{(1)}(\tau)$ is observed for the polariton laser as the condensate occupancy is increased. As we show in Fig. 5(c), just above threshold, at a low condensate occupancy of $\bar{n} \sim 2.7, g^{(1)}(\tau)$ decays exponentially with $\tau$ [Fig. 5(c)], corresponding to a Lorentzian line shape. This confirms single-mode lasing and clearly shows 


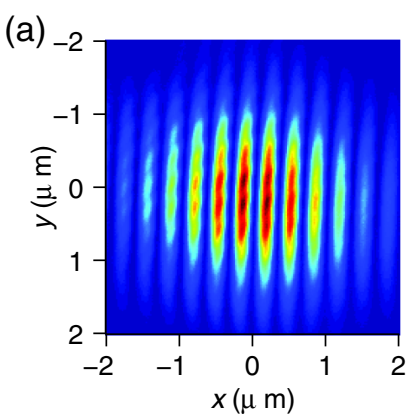

(c)

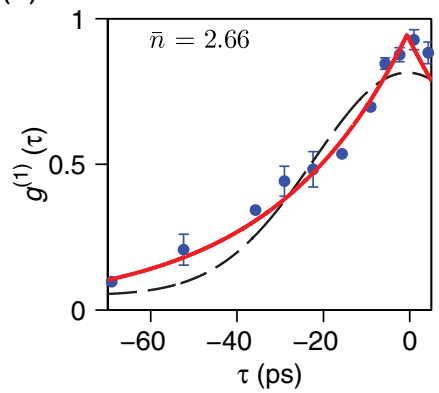

(b)

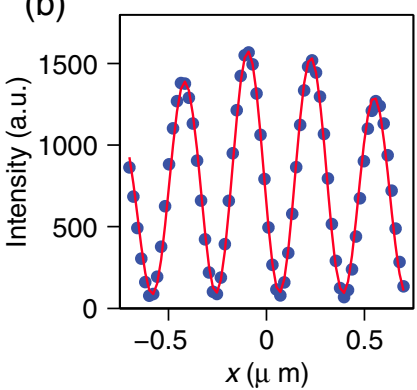

(d)

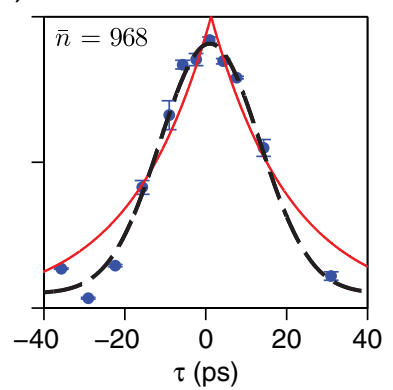

(e)

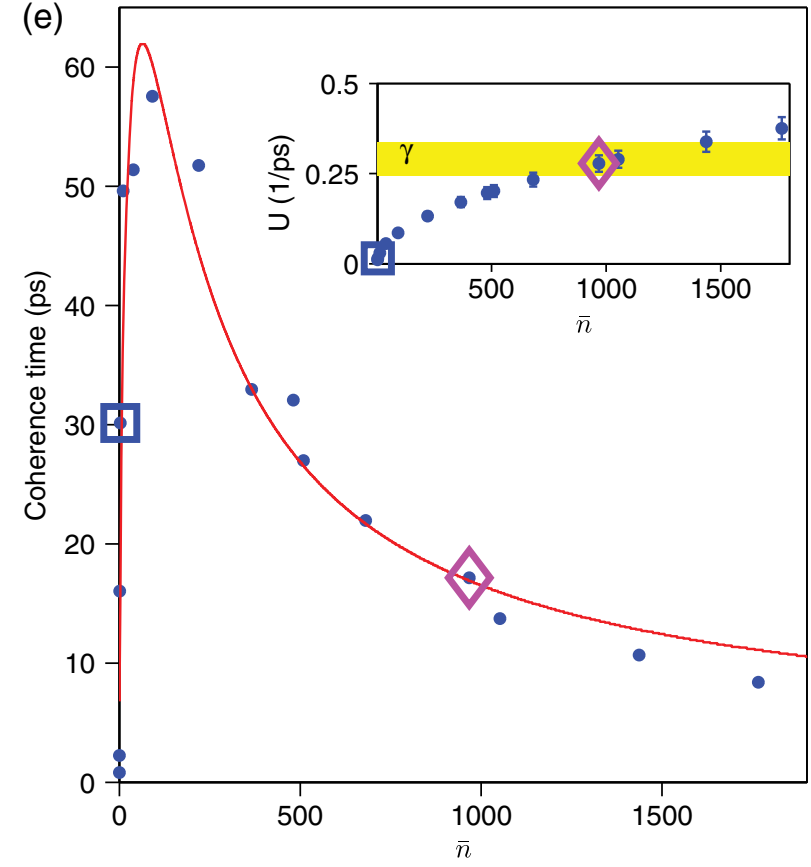

FIG. 5. First-order coherence properties of the single-mode polariton laser. (a) A spatial interference image of the polariton laser at zero time delay. (b) The interference fringe along $x$ axis obtained from (a) by integration along the $y$ axis. The dots are data, and the solid line is a fit by Eq, (A1) with $g^{(1)}(0)=0.94$ (see the Appendix). (c,d) The measured $g^{(1)}(\tau)$ vs $\tau$ (dots), exponential fits (red solid lines), and Gaussian fits (black dashed lines), for $\bar{n}=2.66$ and 968, respectively. (e) The phase coherence time $\tau_{c}$ of the polariton laser vs $\bar{n}$. The dots are taken from the measured $g^{(1)}(\tau)$ for each value of $\bar{n}$. The line is calculated from Eq. (3) using the fitted parameters. Inset: Comparison between the interaction energy $U$ (dots) and decay rate $\gamma$ (yellow-shaded region) of the condensate vs $\bar{n}$. The error bars and thickness of the shaded area are determined by fitting errors with a 95\% confidence interval. The rectangle and diamond marks represent $\bar{n}=2.66$ and $\bar{n}=968$, respectively.

that the intrinsic dephasing of the condensate dominates over external effects. As $\bar{n}$ increases, $g^{(1)}(\tau)$ changes to Gaussian decay, as is apparent in Fig. 5(d) for $\bar{n}=968$. The coherence time also decreases. This decrease cannot be explained by multimode lasing or extrinsic effects, which were excluded by the exponential decay at small $\bar{n}$. It is also distinct from photon lasers, where exponential decay of $g^{(1)}(\tau)$ and the Lorentzian spectrum persists in single-mode lasers.

The transition to a Gaussian decay of $g^{(1)}(\tau)$ can be described using the same single-mode matter-wave laser theory as used to describe the $g^{(2)}$ measurements. The $g^{(1)}(\tau)$ of a matter-wave laser is given by $[32,41]$

$$
\begin{aligned}
\left|g^{(1)}(\tau)\right|= & \exp \left(-\frac{4\left(n_{s}+\bar{n}\right) u^{2}}{\bar{\gamma}^{2}}(\exp (-\bar{\gamma} \tau)+\bar{\gamma} \tau-1)\right) \\
& \times \exp \left(\frac{n_{s}+\bar{n}}{4 \bar{n}^{2}}(\exp (-\bar{\gamma} \tau)-\bar{\gamma} \tau-1)\right), \\
\bar{\gamma}= & \frac{\bar{n}}{n_{s}+\bar{n}} \gamma .
\end{aligned}
$$

Here, $u$ is the polariton-polariton interaction constant and $\gamma$ is the decay rate of the polariton ground state.
This equation can be simplified in limiting cases corresponding to the weak- and strong-interaction regimes. To separate the two regimes, we define a total interaction strength $U=4 u \sqrt{\bar{n}}$. In the weak-interaction regime, $U \ll \gamma$, Eq. (3) is reduced to the Schawlow-Townes formula for a photon laser, $\exp (-\gamma \tau / 2 \bar{n})$, featuring an exponential decay [Fig. 5(c)]. Correspondingly, the $1 / e$ coherence time increases linearly with the occupation number of the lasing mode, $\tau_{c}=2 \bar{n} / \gamma$. In the stronginteraction regime, $U \gg \gamma$, Eq. (3) is approximated by $\exp \left(-2 \bar{n} u^{2} \tau^{2}\right)$, featuring a Gaussian decay, as we observed [Fig. 5(d)]. Correspondingly, $\tau_{c} \propto 1 / U \propto 1 / \sqrt{\bar{n}}$. Therefore, polariton-polariton interactions and the shot noise of the condensate lead to a Gaussian broadening or dephasing of the polariton laser.

Using Eq. (3) to fit the $g^{(1)}(\tau)$ data at different $\bar{n}$, we can obtain the main parameters governing the dynamics of the polariton laser: $n_{s}, \gamma$, and $u$. The best fit yields $n_{s}=$ $61 \pm 13, \gamma=0.29 \pm 0.04 \mathrm{ps}^{-1}$, and $u=(2.2 \pm 0.2) \times 10^{-3} \mathrm{ps}^{-1}$. Here, $n_{s}$ is similar to the estimate obtained from pulsed $g^{(2)}$ measurements, 37.3. The difference may be due to different excitation conditions, CW vs pulsed, which may lead to different densities and effective temperatures of the reservoir. Note that $\gamma$ is within a reasonable range. From $u$, we estimate the system-size-independent interaction constant 
$u A_{\text {cond }}=4 \mu \mathrm{eV} \times \mu \mathrm{m}^{2}$, which is in excellent agreement with the previously reported theoretical and experimental values [42]. Here, $A_{\text {cond }} \sim 2.5 \mu \mathrm{m}^{2}$ is the size of the condensate measured from spatial PL imaging (see the inset of Fig. 1 for an example). It is independent of the pump power, as expected, since it is determined by the effective confinement potential in OD systems. This confirms that the strong polariton-polariton interaction $u$ could be achieved in our system because of the tight lateral confinement or small $A_{\text {cond }}$.

Figure 5(e) compares $\tau_{c}$ from the fit with experimental values with respect to $\bar{n}$. For small $\bar{n}, \tau_{c}$ increases sharply with $\bar{n}$, as expected from the Schawlow-Townes formula. However, $\tau_{c}$ starts to decrease because polariton-polariton scattering leads to the phase decoherence of the lasing mode. The crossover between the weak- and stronginteraction regimes corresponds to where $U \sim \gamma$, as illustrated in the inset of Fig. 5(e).

We note that, although linewidth broadening has been observed in polariton lasers before, the contribution from the condensate nonlinearity is negligible. Typically, the linewidth broadening is accompanied by an increase in the intensity noise $[21,24]$ and thus is understood as the effect of mode competition. When a single, localized mode is selected, the coherence time saturates above threshold and becomes independent of the ground-state occupancy [16]; the coherence time is mainly limited by the energy shift, resulting from reservoir-induced thermal fluctuations represented by $n_{s} \gg \bar{n}$. Here, however, the intensity noise remains at the shot-noise limit, and thus, multimode lasing or reservoir-induced fluctuations are both negligible. In addition, $g^{(1)}(\tau)$ showed strong dependence on the condensate population. Therefore, the Gaussian dephasing we observe directly results from interactions within the condensed polaritons.

We also note that dephasing in the condensate may also be induced by thermal fluctuations of the reservoir population $[16,43]$. However, this effect does not explain the fast decrease of the coherence time above $2 P_{\text {th }}$ and thus is expected to be much weaker compared to the condensate contribution. As shown in Fig. 2(e), the energy shift vs excitation density, $d \Delta E / d P$, between $2 P_{\text {th }}$ and $12 P_{\text {th }}$ is slowed down to $1 / 10$ of that below threshold. Therefore, the energy fluctuation due to reservoir population fluctuation would change by $\ll \sqrt{6}$ between $2 P_{\text {th }}$ and $12 P_{\text {th }}$, which is in direct contradiction to the observed sixfold decrease of the coherence time in this range.

The reservoir-induced fluctuation is suppressed in our system, because the cavity selectively confines the groundstate polaritons, but not the excitons. The energy fluctuation due to particle number fluctuation is proportional to $\sqrt{N} / A$, where $N$ is the particle number and $A$ is the system area. In our system, there is no lateral confinement in the QWs and the excitons can freely diffuse. A typical diffusion length [44] gives a spatial extent of about $100 \mu \mathrm{m}^{2}$. In contrast, the polaritons are tightly confined; their spatial extent is determined by the ground-state wave function and measured to be $2.5 \mu \mathrm{m}^{2}$ for all excitation densities. Hence, the energy fluctuation introduced by an unconfined exciton population is attenuated by 1600 times compared to a confined one. At an exciton density of $0.1 n_{\text {sat }}$, for a saturation density $n_{\text {sat }}=4 \times 10^{10} \mathrm{~cm}^{-2}$ per QW [45], the exciton has a total population of about $4.8 \times 10^{4}$, which introduces an energy fluctuation that is equivalent by about 30 condensate polaritons near zero detuning, while the condensate population quickly increase to $10^{2}-10^{3}$ above threshold. In addition, it would be interesting to investigate if the coherent condensate interacts within itself more strongly than with the thermal reservoir, and if the reservoir-fluctuation-induced dephasing may become suppressed when the coherent condensate is formed [46]. These issues could be clarified in future investigations with more careful calibration of the exciton density or exciton interaction strength.

\section{CONCLUSION}

In conclusion, we have demonstrated the first polariton laser with shot-noise-limited intensity stability, or full second-order coherence, operating as a single-mode laser in the gain saturation regime. At high lasing intensities, we observe a transition from exponential to Gaussian decay of the intrinsic temporal phase coherence of the polariton laser, which can be understood as resulting from strong interactions within the polariton condensate. Experimental results of the phase and intensity noise are well described by an analytical model of single-mode matter-wave lasers, which yield the basic parameters governing a polariton laser's dynamics, including a small gain saturation number and a large polariton interaction strength. The demonstrated intensity stability is a critical feature for lasers. The interaction-induced change in $g^{(1)}(\tau)$ unambiguously reveals the matter-wave origin of the polariton laser. The strong polariton interactions will be important for nonlinear polariton devices [33-35].

\section{ACKNOWLEDGMENTS}

We thank Professor Paul Eastham for helpful discussions. S. K., Z. B., Z. W., and H. D. acknowledge support from the National Science Foundation (NSF) under Grant No. DMR 1150593 and the Air Force Office of Scientific Research under Grant No. FA9550-15-1-0240. C. S., S. B., M. K., and S.H. acknowledge support from the State of Bavaria, Germany. The fabrication of the SWG was performed in the Lurie Nanofabrication Facility (LNF) in Michigan, which is part of the NSF NNIN network.

\section{APPENDIX: MATERIALS AND METHODS}

The sample consists of three stacks of four GaAs/AlAs QWs placed at the central three antinodes of a $\lambda / 2 \mathrm{AlAs}$ cavity. The bottom mirror of the cavity is formed by a DBR 
consisting of 30 pairs of $\mathrm{Al}_{0.15} \mathrm{Ga}_{0.85} \mathrm{As} / \mathrm{AlAs}$ layers. The top mirror of the cavity is formed by an $\mathrm{Al}_{0.15} \mathrm{Ga}_{0.85} \mathrm{As}$ SWG suspended over a three-layer top DBR. The planar wafer is grown by molecular-beam epitaxy. The SWG is created by patterning via electron-beam lithography followed by a reactive-ion etching. Then, a selective wet etching process, followed by critical-point drying, is used to remove an $\mathrm{Al}_{0.85} \mathrm{Ga}_{0.15}$ As sacrificial layer to suspend the grating. We directly measure the energies of the weakly coupled TM excitons and the lower and upper polaritons from their PL, as shown in Fig. 1(b), which gives a Rabi splitting of $12 \mathrm{meV}$ and a detuning of about $0.7 \mathrm{meV}$. The estimated quality factor of the sample from the linewidth measured at low pump power is about 4,000.

The sample is kept at $10 \mathrm{~K}$ and excited by a pulsed or $\mathrm{CW}$ Ti:S laser. The pulsed laser has a pulse width of $150 \mathrm{fs}$ and a repetition rate of $80 \mathrm{MHz}$. The $\mathrm{CW}$ laser is frequencylocked to within a 100-kHz bandwidth and chopped by an electro-optic modulator with $10 \%$ duty cycle at $1 \mathrm{MHz}$ to reduce sample heating. The energy of the pump laser is tuned to at least $20 \mathrm{meV}$ above the exciton resonance to avoid any coherence induced by the pump laser. The $g^{(1)}$ experiments are done using the $\mathrm{CW}$ laser, and the $g^{(2)}$ experiments are done using both the pulsed and $\mathrm{CW}$ lasers. An objective lens is used to focus the pump laser to a spot $2 \mu \mathrm{m}$ in diameter and collect the PL from the sample. The emission is then either sent to a Michelson interferometer or Hanbury Brown-Twiss (HBT) setup for the $g^{(1)}$ and $g^{(2)}$ measurements, respectively.

For $g^{(1)}$ measurements, the intensity distribution in Fig. 5(b) can be described by

$$
\begin{aligned}
I_{\mathrm{CCD}}(\mathrm{x}, \tau)= & I_{1}(x)+I_{2}(x) \\
& +2\left|g^{(1)}(\tau)\right| \sqrt{I_{1}(x) I_{2}(x)} \cos \left(\frac{2 \pi \theta}{\lambda_{0}} x+\phi\right),
\end{aligned}
$$

where $\lambda_{0}$ is the wavelength of the lasing mode, and $\phi, \theta$, and $\tau$ are the phase difference, angle, and time delay between the two interfering beams at a given $\tau . I_{1}(x)$ and $I_{2}(x)$ are the Gaussian intensity profile of the two beams, respectively, and are equal to each other with $<1 \%$ difference in amplitude. Fitting the measured interference patterns with Eq. (A1), we obtain $g^{(1)}(\tau)$ for each $\tau$. Varying the excitation power, we obtain the power dependence of $g^{(1)}(\tau)$ vs $\tau$. We maintain $g^{(1)}(0) \geq 0.9$ throughout the experiments.

[1] R. J. Glauber, The Quantum Theory of Optical Coherence, Phys. Rev. 130, 2529 (1963).

[2] H. Deng, H. Haug, and Y. Yamamoto, Exciton-Polariton Bose-Einstein Condensation, Rev. Mod. Phys. 82, 1489 (2010).
[3] A. Imamoğlu, R. J. Ram, S. Pau, and Y. Yamamoto, Nonequilibrium Condensates and Lasers without Inversion: Exciton-Polariton Lasers, Phys. Rev. A 53, 4250 (1996).

[4] H. Deng, G. Weihs, D. Snoke, J. Bloch, and Y. Yamamoto, Polariton Lasing vs. Photon Lasing in a Semiconductor Microcavity, Proc. Natl. Acad. Sci. U.S.A. 100, 15318 (2003).

[5] S. Christopoulos, G. Baldassarri Höger von Högersthal, A. J. D. Grundy, P. G. Lagoudakis, A. V. Kavokin, J. J. Baumberg, G. Christmann, R. Butt, E. Feltin, J.-F. Carlin, and N. Grandjean, Room-Temperature Polariton Lasing in Semiconductor Microcavities, Phys. Rev. Lett. 98, 126405 (2007).

[6] D. Bajoni, P. Senellart, E. Wertz, I. Sagnes, A. Miard, A. Lemaitre, and J. Bloch, Polariton Laser Using Single Micropillar GaAs-GaAlAs Semiconductor Cavities, Phys. Rev. Lett. 100, 047401 (2008).

[7] S. Kéna-Cohen and S. R. Forrest, Room-Temperature Polariton Lasing in an Organic Single-Crystal Microcavity, Nat. Photonics 4, 371 (2010).

[8] T.-C. Lu, Y.-Y. Lai, Y.-P. Lan, S.-W. Huang, J.-R. Chen, Y.-C. Wu, W.-F. Hsieh, and H. Deng, Room Temperature Polariton Lasing vs. Photon Lasing in a ZnO-Based Hybrid Microcavity, Opt. Express 20, 5530 (2012).

[9] W. Xie, H. Dong, S. Zhang, L. Sun, W. Zhou, Y. Ling, J. Lu, X. Shen, and Z. Chen, Room-Temperature Polariton Parametric Scattering Driven by a One-Dimensional Polariton Condensate, Phys. Rev. Lett. 108, 166401 (2012).

[10] F. Li, L. Orosz, O. Kamoun, S. Bouchoule, C. Brimont, P. Disseix, T. Guillet, X. Lafosse, M. Leroux, J. Leymarie et al., From Excitonic to Photonic Polariton Condensate in a ZnO-Based Microcavity, Phys. Rev. Lett. 110, 196406 (2013).

[11] C. Schneider, A. Rahimi-Iman, N. Y. Kim, J. Fischer, I. G. Savenko, M. Amthor, M. Lermer, A. Wolf, L. Worschech, V. D. Kulakovskii et al., An Electrically Pumped Polariton Laser, Nature (London) 497, 348 (2013).

[12] P. Bhattacharya, B. Xiao, A. Das, S. Bhowmick, and J. Heo, Solid State Electrically Injected Exciton-Polariton Laser, Phys. Rev. Lett. 110, 206403 (2013).

[13] P. Bhattacharya, T. Frost, S. Deshpande, Md.Z. Baten, A. Hazari, and A. Das, Room Temperature Electrically Injected Polariton Laser, Phys. Rev. Lett. 112, 236802 (2014).

[14] H. Deng, G. Weihs, C. Santori, J. Bloch, and Y. Yamamoto, Condensation of Semiconductor Microcavity Exciton Polaritons, Science 298, 199 (2002).

[15] J. Kasprzak, M. Richard, S. Kundermann, A. Baas, P. Jeambrun, J. M. J. Keeling, F. M. Marchetti, M. H. Szymaska, R. André, J. L. Staehli, V. Savona, P. B. Littlewood, B. Deveaud, and L.S. Dang, Boseeinstein Condensation of Exciton Polaritons, Nature (London) 443, 409 (2006).

[16] A. P. D. Love, D. N. Krizhanovskii, D. M. Whittaker, R. Bouchekioua, D. Sanvitto, S. A. Rizeiqi, R. Bradley, M. S. Skolnick, P. R. Eastham, R. André, and L. S. Dang, Intrinsic Decoherence Mechanisms in the Microcavity Polariton Condensate, Phys. Rev. Lett. 101, 067404 (2008).

[17] J. J. Baumberg, A. V. Kavokin, S. Christopoulos, A. J. D. Grundy, R. Butt, G. Christmann, D. D. Solnyshkov, G. Malpuech, G. Baldassarri Höger von Högersthal, E. Feltin, J.-F. Carlin, and N. Grandjean, Spontaneous Polarization 
Buildup in a Room-Temperature Polariton Laser, Phys. Rev. Lett. 101, 136409 (2008).

[18] M. Schellekens, R. Hoppeler, A. Perrin, J. Viana Gomes, D. Boiron, A. Aspect, and C. I. Westbrook, Hanbury Brown Twiss Effect for Ultracold Quantum Gases, Science $\mathbf{3 1 0}$, 648 (2005).

[19] A. Öttl, S. Ritter, M. Köhl, and T. Esslinger, Correlations and Counting Statistics of an Atom Laser, Phys. Rev. Lett. 95, 090404 (2005).

[20] R. G. Dall, S. S. Hodgman, A. G. Manning, M. T. Johnsson, K. G. H. Baldwin, and A. G. Truscott, Observation of Atomic Speckle and Hanbury Brown-Twiss Correlations in Guided Matter Waves, Nat. Commun. 2, 291 (2011).

[21] J. Kasprzak, M. Richard, A. Baas, B. Deveaud, R. André, J. P. Poizat, and L. S. Dang, Second-Order Time Correlations within a Polariton Bose-Einstein Condensate in a CdTe Microcavity, Phys. Rev. Lett. 100, 067402 (2008).

[22] T. Horikiri, P. Schwendimann, A. Quattropani, S. Höfling, A. Forchel, and Y. Yamamoto, Higher Order Coherence of Exciton-Polariton Condensates, Phys. Rev. B 81, 033307 (2010).

[23] M. Aßmann, J.-S. Tempel, F. Veit, M. Bayer, A. RahimiIman, A. Loffler, S. Höfling, S. Reitzenstein, L. Worschech, and A. Forchel, From Polariton Condensates to Highly Photonic Quantum Degenerate States of Bosonic Matter, Proc. Natl. Acad. Sci. U.S.A. 108, 1804 (2011).

[24] J.-S. Tempel, F. Veit, M. Aßmann, L. E. Kreilkamp, A. Rahimi-Iman, A. Löffler, S. Höfling, S. Reitzenstein, L. Worschech, A. Forchel, and M. Bayer, Characterization of Two-Threshold Behavior of the Emission from a GaAs Microcavity, Phys. Rev. B 85, 075318 (2012).

[25] M. Amthor, S. Weienseel, J. Fischer, M. Kamp, C. Schneider, and S. Höfling, Electro-optical Switching between Polariton and Cavity Lasing in an InGaAs Quantum Well Microcavity, Opt. Express 22, 31146 (2014).

[26] K. Kusudo, N. Y. Kim, A. Löffler, S. Höfling, A. Forchel, and Y. Yamamoto, Stochastic Formation of Polariton Condensates in Two Degenerate Orbital States, Phys. Rev. B 87, 214503 (2013).

[27] M. Wouters and V. Savona, Stochastic Classical Field Model for Polariton Condensates, Phys. Rev. B 79, 165302 (2009).

[28] H. Haug, T. D. Doan, H. T. Cao, and D. B. Tran Thoai, Temporal First- and Second-Order Correlations in a Polariton Condensate, Phys. Rev. B 85, 205310 (2012).

[29] P. Schwendimann, A. Quattropani, and D. Sarchi, Stationary and Time-Dependent Correlations in Polariton Condensates, Phys. Rev. B 82, 205329 (2010).

[30] A. L. Schawlow and C. H. Townes, Infrared and Optical Masers, Phys. Rev. 112, 1940 (1958).

[31] H. M. Wiseman, Defining the (Atom) Laser, Phys. Rev. A 56, 2068 (1997).
[32] L. K. Thomsen and H. M. Wiseman, Atom-Laser Coherence and Its Control via Feedback, Phys. Rev. A 65, 063607 (2002).

[33] A. Verger, C. Ciuti, and I. Carusotto, Polariton Quantum Blockade in a Photonic Dot, Phys. Rev. B 73, 193306 (2006).

[34] T. C. H. Liew, A. V. Kavokin, and I. A. Shelykh, Optical Circuits Based on Polariton Neurons in Semiconductor Microcavities, Phys. Rev. Lett. 101, 016402 (2008).

[35] T. C. H. Liew and V. Savona, Single Photons from Coupled Quantum Modes, Phys. Rev. Lett. 104, 183601 (2010).

[36] M. C. Y. Huang, Y. Zhou, and C. J. Chang-Hasnain, A Surface-Emitting Laser Incorporating a High-IndexContrast Subwavelength Grating, Nat. Photonics 1, 119 (2007).

[37] S. J. Schablitsky, L. Zhuang, R. C. Shi, and S. Y. Chou, Controlling Polarization of Vertical Cavity Surface Emitting Lasers Using Amorphous Silicon Subwavelength Transmission Gratings, Appl. Phys. Lett. 69, 7 (1996).

[38] B. Zhang, Z. Wang, S. Brodbeck, C. Schneider, M. Kamp, S. Höfling, and H. Deng, Zero-Dimensional Polariton Laser in a Subwavelength Grating-Based Vertical Microcavity, Light Sci. Appl. 3, e135 (2014).

[39] B. Zhang, S. Brodbeck, Z. Wang, M. Kamp, C. Schneider, S. Höfling, and H. Deng, Coupling Polariton Quantum Boxes in Sub-Wavelength Grating Microcavities, Appl. Phys. Lett. 106, 051104 (2015).

[40] See Supplemental Material at http://link.aps.org/ supplemental/10.1103/PhysRevX.6.011026 for estimation of upper and lower bounds of measured $g^{(2)}(0)$.

[41] D. M. Whittaker and P. R. Eastham, Coherence Properties of the Microcavity Polariton Condensate, Europhys. Lett. 87, 27002 (2009).

[42] L. Ferrier, E. Wertz, R. Johne, D. D. Solnyshkov, P. Senellart, I. Sagnes, A. Lemaitre, G. Malpuech, and J. Bloch, Interactions in Confined Polariton Condensates, Phys. Rev. Lett. 106, 126401 (2011).

[43] A. Kavokin, J. J. Baumberg, G. Malpuech, and F. P. Laussy, Microcavities (Oxford University Press, New York, 2007).

[44] G. Tosi, G. Christmann, N. G. Berloff, P. Tsotsis, T. Gao, Z. Hatzopoulos, P. G. Savvidis, and J. J. Baumberg, Sculpting Oscillators with Light within a Nonlinear Quantum Fluid, Nat. Phys. 8, 190 (2012).

[45] R. Houdré, J. L. Gibernon, P. Pellandini, R. P. Stanley, U. Oesterle, C. Weisbuch, J. O'Gorman, B. Roycroft, and M. Ilegems, Saturation of the Strong-Coupling Regime in a Semiconductor Microcavity: Free-Carrier Bleaching of Cavity Polaritons, Phys. Rev. B 52, 7810 (1995).

[46] H. Haug, H. T. Cao, and D. B. Tran Thoai, Coherence and Decoherence of a Polariton Condensate, Phys. Rev. B 81, 245309 (2010). 